\title{
Pro-active Page Replacement for Scientific Applications: A Characterization
}

\author{
Murali Vilayannur Anand Sivasubramaniam Mahmut Kandemir \\ 111 I.S.T Building \\ Department of Computer Science and Engineering \\ Pennsylvania State University, University Park, PA 16802, USA \\ \{vilayann, anand, kandemir\}@cse.psu.edu
}

Phone: 814-863-3627

\begin{abstract}
Paging policies implemented by today's operating systems cause scientific applications to exhibit poor performance, when the application's working set does not fit in main memory. This has been typically attributed to the sub-optimal performance of LRU-like virtual-memory replacement algorithms. On one end of the spectrum, researchers [MDK96, BM00] in the past have proposed fully automated compiler-based techniques that provide crucial information on future access patterns (reuse-distances, release hints etc) of an application that can be exploited by the operating system to make intelligent prefetching and replacement decisions. Static techniques like the aforementioned can be quite accurate, but require that the source code be available and analyzable. At the other end of the spectrum, researchers [SKW99, $\mathrm{LCC}^{+}$97, GC97] have also proposed pure system-level algorithmic innovations to improve the performance of LRU-like algorithms, some of which are only interesting from the theoretical sense and may not really be implementable. Instead, in this paper we explore the possibility of tracking application's runtime behavior in the operating system, and find that there are several useful characteristics in the virtual memory behavior that can be anticipated and used to pro-actively manage physical memory usage. Specifically, we show that LRU-like replacement algorithms hold onto pages long after they outlive their usefulness and propose a new replacement algorithm that exploits the predictability of the application's page-fault patterns to reduce the number of page-faults. Our results demonstrate that such techniques can reduce page-faults by as much as $78 \%$ over both LRU and EELRU [SKW99] that is considered to be one of the state-of-the-art algorithms towards addressing the performance shortcomings of LRU. Further, we also present an implementable replacement algorithm within the operating system, that performs considerably better than the Linux kernel's replacement algorithm.
\end{abstract}

Keywords: Virtual Memory, Predictive Page Replacement, LRU, EELRU 


\section{Introduction}

Many scientific applications of today manipulate problems that access large data sets. In general, these data sets are disk-resident and their sizes range from mega-bytes to tera-bytes. These include applications from medical imaging, data analysis, video processing, large archive maintenance, space telemetry data, and so on. Typically, such applications are explicitly coded to stage the data to be manipulated from disk or the in-core versions of the same program is scaled to handle the larger problem sizes. It has been well-documented [SKW99] that LRU-like virtual memory replacement algorithms under-perform for typical scientific applications that tend to cyclically access pages of memory. Consequently, a paged virtual memory system (or the scaled version of the in-core program) is not considered a viable option [WGRW93] for solving out-of-core problems. When virtual memory pages are touched cyclically and the working set is slightly larger than available physical memory, LRU chooses to evict exactly the page that would be referenced the soonest. In the above scenario, the optimal algorithm would evict those pages that were referenced recently since it has future knowledge of the reference patterns. The basic idea behind our work is as follows - if we can predict or estimate the lifetimes of all the memory-resident pages, then we could evict a page as soon as we know that there would be no more references to that page. In a sense, what we are trying to accomplish here is an online-approximation of the optimal algorithm which always evicts the pages that would be referenced the furthest in time. The basic motivation behind our idea is that the LRU replacement algorithm holds onto pages that have long been dead, and hence any replacement algorithm should be pro-active instead of being reactive, and anticipate candidates for eviction. In practice, however, it is quite difficult to accurately predict future access information and lifetimes of virtual memory pages. Consequently, the algorithm that we propose uses past access pattern information as an indicator to predict lifetime distances of pages. In this regard, we have experimented with a few different predictors and compared and evaluated their performance for a set of twelve-different memory-intensive applications drawn from the NAS parallel and SPEC 2000 benchmark suites. We now introduce a few notations and definitions that will be used later on in the text:

- Denote the distance between the first access (touch) and the last access (touch) for a virtual memory page before which it gets replaced as the lifetime distance $(\mathbf{L})$

- Denote the distance between the last access (touch) and the subsequent reuse access to a virtual memory page during which time the page was replaced as the reuse distance $(\mathbf{R})$

- Denote the distance between the replacement of a page and the subsequent reuse access to the same virtual memory page as the window distance $(\mathbf{W})$

- Denote the distance between the last access (touch) and the replacement of the page as the dead-page distance (D)

- Denote the distance between two successive page faults to a virtual memory page as the page-fault distance (F)

These notations are pictorially shown in Figure 1. Henceforth, we shall refer to these parameters as the page-fault parameters or simply the fault parameters. Note that in the above definitions, the notion of distance was deliberately left undefined since it could be measured in many different ways (for example in terms of memory references, number of page faults to other pages, number of references to unique memory pages or perhaps even time). Ideally, we would like to measure these distances in terms of memory references to unique pages since that could directly translate to whether or not a page would be retained for a given memory configuration (this is similar to the idea that was proposed in [JZ02] in the context of buffer caches), which is fairly difficult if not impossible to do on an actual system since that would involve an unacceptable overhead of trapping on each and every memory reference, or would require special hardware support (like an augmented MemorIES [NMS $\left.{ }^{+} 00\right]$ board) to store the timestamps of last access to pages. Without hardware support, since the only OS-visible events are page-faults and replacements, it is possible to measure $\mathbf{W}$ and $\mathbf{L}+\mathbf{D}$ alone, and that too only in terms of units that are visible to the OS ( eg. in terms of the number of page-faults incurred by other pages). Each virtual memory page (p) is thus characterized by a unique 4-tuple $t_{i}^{p}=$ $(\mathbf{L}, \mathbf{R}, \mathbf{D}, \mathbf{W})$ between page faults $i$ and $i+1$. Consequently, a program's execution can be visualized as a sequence of such tuples. The objectives of this paper are as follows:

- Show that LRU-like replacement algorithms hold onto pages that have long been dead, thus losing opportunities for reducing page-faults. 


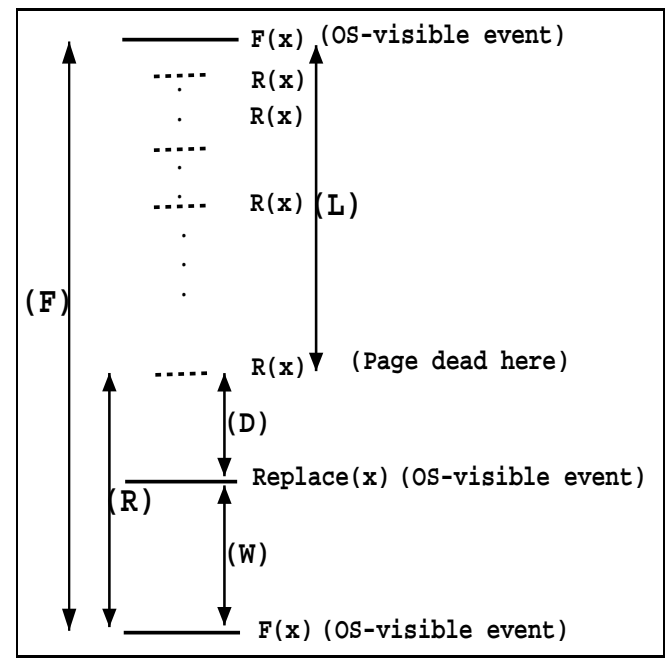

Figure 1: Page-fault characterization.

- Characterize the variations of some of these parameters $(\mathbf{L}, \mathbf{D})$ in terms of memory references and in terms of page faults and see if there is any kind of correlation and predictable patterns between these, and

- Use these parameters in conjunction with simple predictors to design application-specific replacement algorithms.

If we can predict $\mathbf{L}$ values, then we can evict pages earlier than when an operating system might choose to evict them, because a virtual memory page typically becomes dead much before the system's replacement algorithm (like LRU) decides to evict the page. However, an incorrect prediction of $\mathbf{L}$ may degrade the performance of the application by increasing the number of page-faults incurred by the application. Ideally, we would like the predicted value of $\mathbf{L}$ to be as large as possible, but not larger than $\mathbf{L}+\mathbf{D}$. Likewise, if we can predict $\mathbf{W}$ values, we could prefetch pages well-ahead of when they would be actually referenced. However, an incorrect prediction of $\mathbf{W}$ could also degrade performance by increasing number of page-faults incurred by applications. Ideally, we would like the predicted value of $\mathbf{W}$ to be as large as possible such that the sum of the predicted value and the time it takes to fetch the data from disk/peripheral device is no larger than W. Consequently, this work can be considered as an applicationcustomized prefetching and replacement technique that is done automatically and transparently at runtime by the operating system, and is hence more generally applicable than what was proposed in [MDK96]. In this paper, we only explore the predictability characteristics of the lifetime distances for effective page replacement and leave predictive prefetching as a future extension.

The rest of this paper is organized as follows. Section 2 discusses related work. Section 3 describes the experimental setup and the scientific applications that we used as benchmarks for characterization and evaluation. In Section 4, we illustrate page-fault characteristics of the system replacement algorithm. Section 5 proposes a new replacement algorithm and evaluates its performance. We finally conclude and discuss the scope for future work in Section 6.

\section{Related Work}

Over the last few decades, a lot of work [Cor69, OOW93, JS94, SKW99, LCC ${ }^{+}$97, GC97, PG95, JZ02] has been done to address the shortcomings of LRU-like replacement algorithms. Some of these [SKW99, OOW93, LCC ${ }^{+}$97, GC97] try to address the shortcomings for a specific workload access pattern such as cyclical access patterns of programs whose working set sizes are larger than available physical memory. In [SKW99], the authors propose an adaptive replacement algorithm (EELRU) that uses the same kind of recency information that is normally available to LRU and a simple online cost-benefit analysis to guide its replacement decisions. In their approach, the system continuously monitors the performance of the LRU algorithm and if it detects the worst-case behavior, it tries to pro-actively evict 
pages. Our work in this paper is similar to theirs in the sense that we wish to evict pages early if we detect that this could do better than LRU, but is different from theirs in the methodology that we employ to detect such situations. In the context of buffer-caches where the system gets control for every reference/access, researchers have proposed adaptive variants of LRU such as [OOW93, $\mathrm{LCC}^{+}$97] and our work is inherently different from theirs because of the different domains of applicability. In [GC97], the authors propose a new replacement algorithm (SEQ) that detects a long sequence of page-faults and resorts to Most-Recently-Used replacement algorithm on detecting such sequences. In spirit, most of the proposed algorithms try to imitate the behavior of the OPT [Bel66] algorithm and therein lies the similarity of our proposed work with them. In [PG95], the authors propose a $k$-order Markov chain to model the sequence of time intervals between successive references to the same address in memory during program execution. Note that in this work, we are only interested in the sequence of time intervals between successive page faults to the same virtual memory page. In a more recent work [JZ02], the authors propose an efficient buffer cache replacement policy called LIRS (Low Interference Recency Set) that uses recency to evaluate Inter-Reference Recency for making a replacement decision. The key insight of this technique is that it attempts to capture the inter-reference recency (the number of unique blocks accessed between two consecutive references to a block) to overcome the deficiencies of LRU. In [MM04], the authors propose a self-tuning, low overhead, scan resistant algorithm (ARC) that exploits both recency and frequency to adapt itself according to the workload characteristics. A modified version of the algorithm (CAR), that is more amenable for virtual memory replacement, was proposed in a more recent paper [BM04] that combines the benefits of ARC [MM04] and CLOCK [Cor69]. Both ARC and CAR require significant amount of memory for recording history information that could prove to be expensive for memory intensive applications considered in this paper. Additionally, many of the proposed algorithms in the literature are usually interesting only from the theoretical point of view and may not really be implementable in an operating system. In this work, we also demonstrate a potential in-kernel approximation of our idea.

From a system's perspective, a lot of work has been proposed [CG99, FC03, CFL94, PGG ${ }^{+}$95b] for applications that access disk-resident data sets through explicit I/O invocations. In the past, researchers have also studied the problem of poor virtual memory performance (implicit I/O) from an application's perspective [BM00, MDK96, HC92, CE97]. Researchers in [MDK96] propose automatic compiler-driven techniques to modify application codes to prefetch memory pages from disk/peripheral I/O devices. In their prefetching scheme, the compiler provides the crucial information on future access patterns, the operating system provides a simple interface for prefetching, and the run-time accelerates performance by adapting to runtime behavior. In a subsequent work [BM00], they show that primitives to release memory pages that are no longer used (if used judiciously) when used in conjunction with their prefetching schemes improves the response times of concurrently running interactive tasks. However, static techniques like the above require that the source code be available and analyzable. On the OS side, a lot of work has been done towards detection of file access patterns automatically in the file system [GA94, KL96] or parametric specification of file access patterns supplied by the application $\left[\mathrm{PGG}^{+}\right.$95a, CFKL95]. However, much of this work involves using explicit $\mathrm{I} / \mathrm{O}$ interfaces to stage data from peripheral devices.

On the compiler side, researchers have primarily looked at reordering computation to improve data reuse and reduce I/O time [BCR96] or inserting explicit I/O calls into array-based codes [PKK95, BTC94]. Typically, compilers are aided by annotated source code or programming language extensions to indicate properties of important data structures. Amongst the techniques developed to improve I/O performance of applications by predicting reuse-distances and dead-page distances, the closest work is by Mowry et. al in [BM00, MDK96]. However, the method that we propose is a pure runtime technique and does not require any source-level modifications. On the hardware side, researchers have proposed a dead-block prediction scheme in [LFF01] that predicts when a cache block becomes "dead" and hence evictable. While our work shares a common goal with theirs, prediction of lifetimes of virtual memory pages is inherently a different problem.

\section{Experimental Framework}

We now describe the applications and the simulation platform that we use in this study. 


\subsection{Applications}

To evaluate the effectiveness of our approach, we measured its impact on the performance of a selected set of memory intensive SPEC CPU 2000 workloads [Hen00] and seven of the memory-intensive sequential versions of the NAS Parallel benchmark (NPB) suite $\left[\mathrm{BBB}^{+} 91\right]$. Note that the benchmarks that we have used in the paper are amongst the most memory intensive applications of the SPEC benchmark suite. There is no inherent difficulty in running other SPEC benchmarks, but most of the other SPEC benchmarks are CPU intensive and their working set sizes are very small and consequently do not stress the virtual memory subsystem at all. It is for the same reason, that we also show results for 7 of the 8 NAS benchmarks whose virtual memory footprints are fairly large in addition to the SPEC applications.

All the $\mathrm{C}$ benchmarks were compiled with gec version 3.2.2 at an optimization level -O3, and the Fortran 90 benchmarks were compiled with the Intel Fortran 90 compiler at the same optimization level. A brief description of the benchmarks and the sizes of the data sets that they access are shown in Table 1. Since different applications have different working set sizes, and since we wanted to exercise the virtual memory capabilities of the system, we configured the memory available differently for these applications. The specific values are given in Table 1. Unless, otherwise mentioned the memory configuration that we simulated for the characterization experiments was fixed at $300 \mathrm{MB}$ for most of the NAS Parallel Benchmarks, 128 MB for LU, CG and all SPEC 2000 workloads with the exception of GZIP and MCF for which we fix it at $64 \mathrm{MB}$.

Table 1: Description of applications: The Total Memory column indicates the total/maximum memory that is used by the application, and the Simulated Memory column indicates the simulated memory size that was used for the characterization.

\begin{tabular}{|c|c|c|c|c|}
\hline Name & Description & Input Data Set & $\begin{array}{l}\text { Total } \\
\text { Memory }\end{array}$ & $\begin{array}{l}\text { Simulated } \\
\text { Memory }\end{array}$ \\
\hline IS & Integer Bucket Sort & $2^{25}$ 21-bit integers & $384 \mathrm{MB}$ & $300 \mathrm{MB}$ \\
\hline CG & $\begin{array}{l}\text { Conjugate Gradient Method to } \\
\text { solve an unstructured sparse-matrix }\end{array}$ & $\begin{array}{l}75000 \times 75000 \text { sparse matrix } \\
\text { with } 15825000 \text { non-zeroes }\end{array}$ & $399 \mathrm{MB}$ & $128 \mathrm{MB}$ \\
\hline FT & 3-D Fast-Fourier Transform & $\begin{array}{l}256 \times 256 \times 256 \text { matrix } \\
\text { of complex numbers }\end{array}$ & $584 \mathrm{MB}$ & $300 \mathrm{MB}$ \\
\hline MG & 3-D Multi-Grid Solver & $256 \times 256 \times 256$ matrix & $436 \mathrm{MB}$ & $300 \mathrm{MB}$ \\
\hline SP & Diagonalized Approximate Factorization & $102 \times 102 \times 102$ matrices & $323 \mathrm{MB}$ & $300 \mathrm{MB}$ \\
\hline BT & Block Approximate Factorization & $5 \times 5 \times 65 \times 65 \times 65$ matrices & $400 \mathrm{MB}$ & $300 \mathrm{MB}$ \\
\hline $\mathrm{LU}$ & Simulated CFD using SSOR techniques & $5 \times 102 \times 102$ matrices & $178 \mathrm{MB}$ & $128 \mathrm{MB}$ \\
\hline GZIP & GNU Compression Utility & $\begin{array}{l}\text { TIFF Image, Webserver log, } \\
\text { binary, random data, tarball }\end{array}$ & $192 \mathrm{MB}$ & $64 \mathrm{MB}$ \\
\hline WUPWISE & Physics/Quantum Chromodynamics & NITER $=75, \mathrm{KAPPA}=2.4 \mathrm{E}-1$ & $176 \mathrm{MB}$ & $128 \mathrm{MB}$ \\
\hline SWIM & Shallow Water Modeling & $1335 \times 1335$ matrix, 200 iterations & $190 \mathrm{MB}$ & $128 \mathrm{MB}$ \\
\hline MCF & Combinatorial Optimization & default input & $190 \mathrm{MB}$ & $64 \mathrm{MB}$ \\
\hline APSI & Meteorology & $\begin{array}{l}112 \times 112 \times 112 \text { matrix } \\
\text { for } 70 \text { iterations }\end{array}$ & $191 \mathrm{MB}$ & $128 \mathrm{MB}$ \\
\hline
\end{tabular}

\subsection{Experimental Platform}

We characterize the virtual memory behavior of these applications and the potential of our proposed replacement algorithms in the context of an execution-driven x86 simulator. The simulations were executed on a Linux-2.4.20 kernel on a dual 2.7 GHZ Xeon workstation with a total of $1 \mathrm{~GB}$ physical memory and a 36 GB SCSI disk. The execution-driven simulator that we used in this study is valgrind [SN03] which is an extensible x86 memory debugger and emulator. Valgrind is a framework that allows for custom skins/plugins to be written that can augment the basic blocks of the program as it executes. The skins/plugins that we implemented augmented the basic blocks to return control to the skin after every memory-referencing instruction with the value of the memory address that was referenced. The skins maintain data structures necessary for implementing the techniques that we will be describing shortly and for collecting the relevant statistics. The page fault statistics for these applications that were used for comparison with the kernel-implementable version of our scheme were obtained on a uni-processor Xeon workstation running the 2.4.20 Linux kernel. 


\section{Characterization Results}

We first take a look at the page-fault characteristics of these applications. As indicated in the previous section, we would like to characterize an application's execution based on the fault parameters. In particular, we want to understand the reasons as to why LRU performs poorly from the fault parameters perspective, i.e, we want to characterize those situations in which LRU holds on to the page long after it has been "dead". To illustrate this, we give the cumulative distribution plot of the ratio $\mathbf{D} /(\mathbf{L}+\mathbf{D})$ for the LRU replacement algorithm for all the applications for a specific memory configuration. If the replacement algorithm is really doing a good job, then it would replace a page as soon as it became dead, or in other words, all replaced pages would have a small dead-time distance $\mathbf{D}$. In order to normalize the notion of small, we compute the ratio $\mathbf{D} /(\mathbf{L}+\mathbf{D})$ and plot the cumulative distribution plot of this ratio. Based on the above explanation, we can now interpret the cumulative distribution plot of this ratio as follows - if the plot peaks early, then the algorithm does a good job of evicting pages as soon as they become dead. On the other hand, if it peaks late, then the algorithm is not doing a good job.

In this study, we have used the following units to measure the distance - number of memory references and number of page-faults to other pages. The reason we plotted two sets of graphs was because the former is a quantity that needs hardware support and can only be approximated in practice, while the latter can be measured by the operating system. In Figures 2 (a) and 3 (a), we find that with the exception of BT, LU and WUPWISE, the replacement algorithm is not evicting dead pages quickly since we find that only $15-25 \%$ of all replaced pages have their $\mathbf{D} /(\mathbf{L}+\mathbf{D})$ ratios less than 50\% when distances are measured as number of memory references. Similarly, if we observe Figures 2 (b) and 3 (b), we find that $40 \%$ of all replaced pages have their $\mathbf{D} /(\mathbf{L}+\mathbf{D})$ ratios less than $50 \%$ when the distances are measured as page-faults to other pages. This serves as a motivation for designing a better replacement algorithm that needs to be more pro-active in choosing candidates for eviction. Thus, it is clear that the LRU replacement algorithm performs poorly from the fault parameters perspective. We next study the predictability characteristics of the fault parameters before we describe the proposed algorithm.

(a)

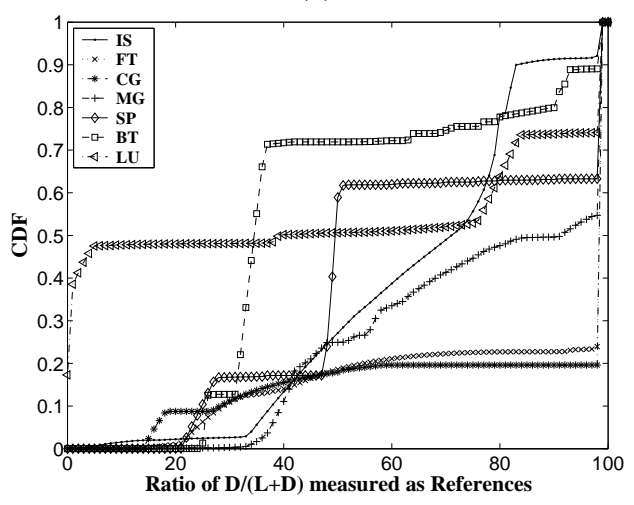

(b)

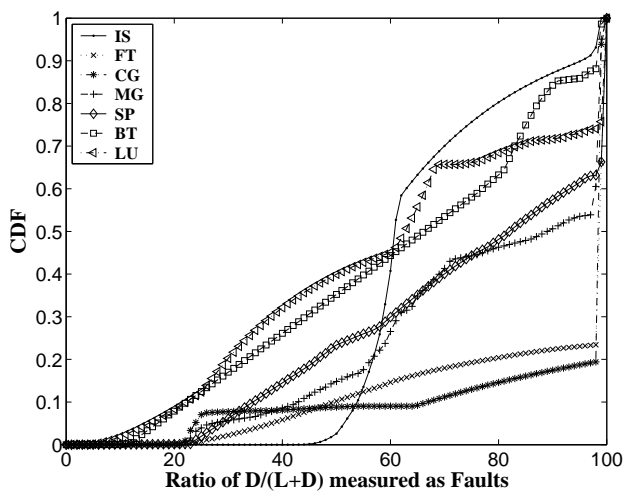

Figure 2: Ratio of D/L+D measured for NPB as (a) References, (b) Faults.

As was stated earlier, a pro-active replacement algorithm should evict a page as soon as it becomes "dead". However, in order for the system to predict which pages will become dead the soonest, it needs to predict the lifetime distances (L) of pages. Hence, we felt that characterizing the variability and hence predictability of the lifetime distance (L) was necessary, and may even provide insights into the design of the predictors for the replacement algorithm. All the characterization experiments were conducted using exact LRU as the replacement algorithm.

The first characterization experiment plots the cumulative distribution of the absolute differences between successive values of lifetime distances of a virtual memory page. It is expected that if lifetime distances are similar, the successive differences would be close to zero and hence a CDF plot of the frequencies of occurrence of the differences would be steep in the beginning. Yet another metric that can be gleaned from such a plot is the number of different dominant values for the distribution. In this experiment, we compute the frequency of occurrences for differences up-to a large value (set to 50000), and all the differences greater than that are treated identically. Yet another property that we wished to look at was whether the magnitude of the differences had any discernible characteristic that we could take advantage of. A cumulative distribution plot of the frequencies of occurrences of differences in lifetime distances 
(a)

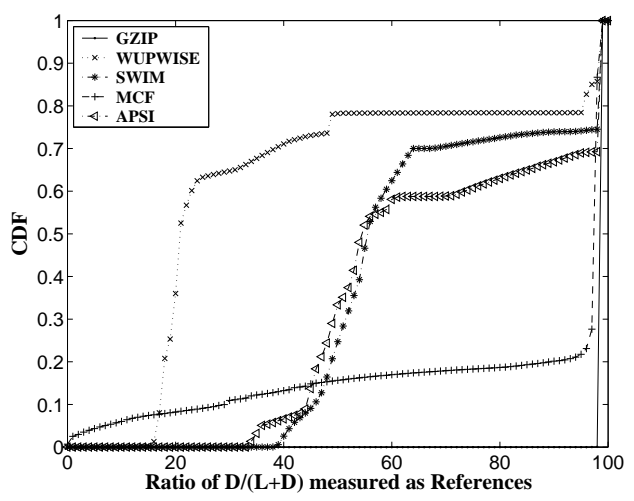

(b)

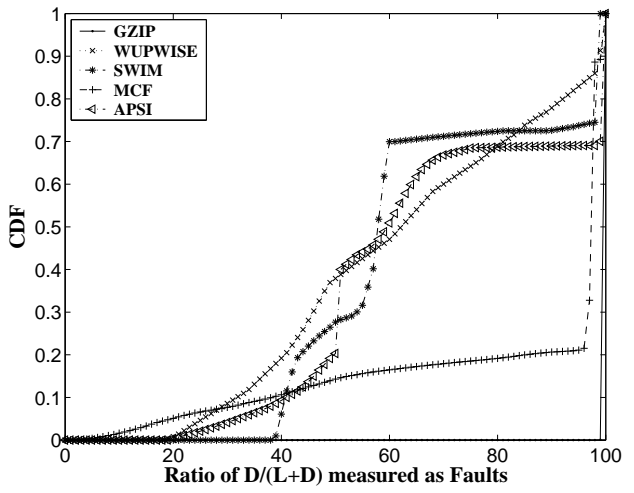

Figure 3: Ratio of D/L+D measured for SPEC2000 as (a) References, (b) Faults.

(absolute difference) is shown in Figures 4 (a) and (b) for the NAS benchmarks, when measured in terms of (a) total memory references, (b) total number of page-faults to other virtual memory pages. Figures 4 (c) and (d) plot the same for the SPEC 2000 benchmarks. Figure 4 (a) indicates that for two of the NAS applications (MG, FT), less than $50 \%$ of occurrences have successive differences less than 10, while all the remaining applications' successive lifetime differences, when measured as memory references, are fairly predictable. Similarly, Figure 4 (b) indicates that with the exception of the same two applications, all the remaining applications have a fairly predictable differences of lifetime distances when measured in terms of page faults. On the other hand, Figure 4 (c) indicates that four of the five SPEC 2000 applications have less than 50\% occurrences of differences of less than 10 when the lifetime distances are measured in terms of memory references, and Figure 4 (b) indicates that two of the five SPEC 2000 applications have less than $55 \%$ occurrences of differences of less than 10 when lifetime distances are measured in terms of page-faults. However, a CDF plot dilutes temporal information and hence cannot be used for determining whether predictability exists or not. Our subsequent experiments indicate that there is sufficient predictability that can be exploited. Figures 5 (a), (b) and Figures 5 (c), (d) plot the same with the only difference being that instead of the absolute value, the actual difference between the successive lifetime distances is used for the NAS and SPEC benchmarks respectively. From both these plots, it is clear that for most of the benchmarks, differences of successive lifetime distances are fairly symmetric on either side of zero, and a majority of them lie within a bounded range, which we surmise is because of the applications' structured access patterns. Figures 6 (a), (b) and Figures 6 (c), (d) plot the cumulative distribution of the absolute differences between successive $(\mathbf{L}+\mathbf{D})$ values of a virtual memory page. Note that a realizable implementation of any replacement algorithm in the operating system cannot rely on accurately tracking memory references to calculate lifetime distances. Therefore, the operating system must rely upon pagefaults and page replacement events to approximate lifetime distances of pages, or in other words the operating system needs to approximate the lifetime distance using the measured $\mathbf{L}+\mathbf{D}$ distances. It can be observed that the CDF plots in Figures 6 (a), (b) and Figures 6 (c), (d) largely mirror the distribution of the lifetime distances' differences, though the exact values tend to differ.

In the second set of experiments, we plot the cumulative distribution of the lifetime distance measured both in terms of the number of references and in terms of the number of page-faults to other pages as shown in Figures 7 (a) and (b), respectively (for the same reasons as stated before due to the inability of the operating system to measure distances in terms of number of memory references without hardware support). Although these graphs indicate that there are no dominant values of lifetime distances and a seeming lack of predictability, it should be kept in mind that a CDF plot dilutes and hides temporal information. Suppose, we consider a highly predictable sequence $1,2,3,1,2,3$.. and so on, a cumulative distribution plot would be a $45^{\circ}$ straight-line since each unique value in the sequence occurs with equal frequency.

Towards determining whether sufficient predictability exists in the sequence of lifetime distances, we plot the variation of lifetime distances with time in our next set of experiments. It is to be noted here that while such a characterization is relevant only for a fixed deterministic replacement algorithm (eg. exact LRU) and a particular memory configuration, it is important to see if there are any patterns that can be exploited. In the interest of space, we show the time plots only for a few applications. While there seems to be a certain degree of regularity and hence predictability in the variation of $\mathrm{L}$ with time as seen in Figures 8 (a), (b) and 9 (a), (b), this trend is not immediately evidenced in the cumulative 
(a)

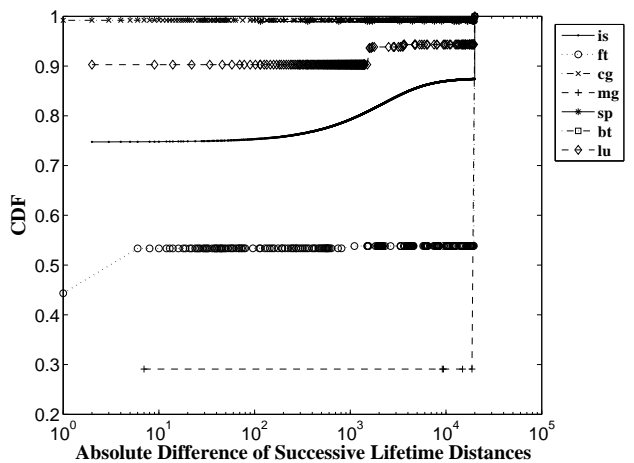

(c)

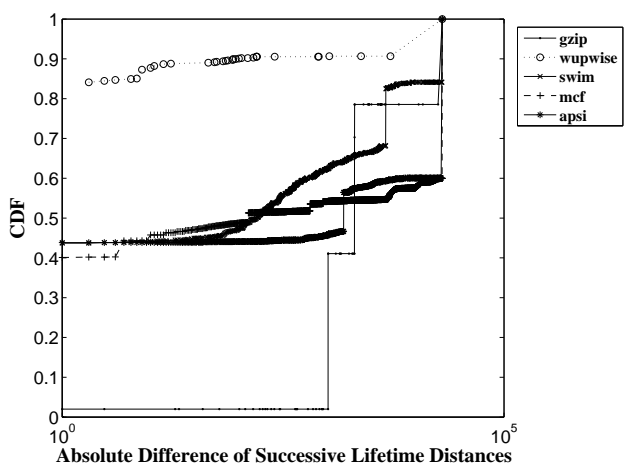

(b)

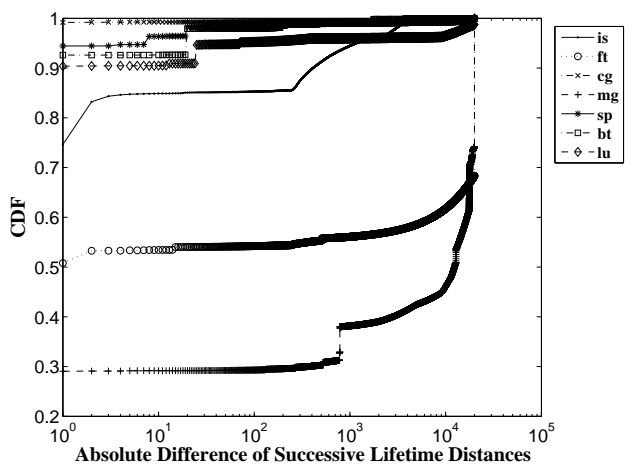

(d)

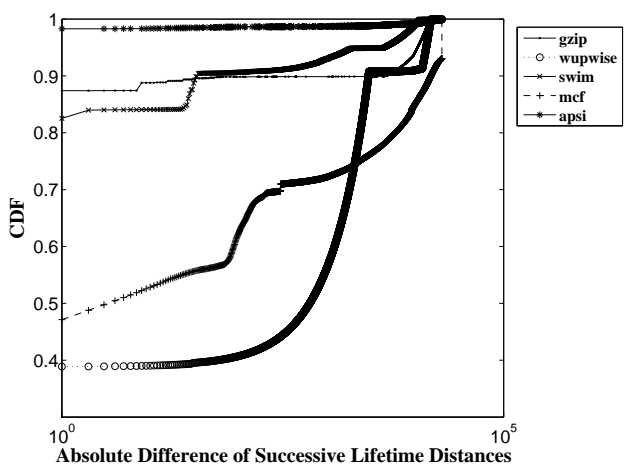

Figure 4: Absolute differences between successive L distances measured as (a) NPB2.3 - Total Memory References, (b) NPB2.3 - Faults to other pages, (c) SPEC2000 - Total Memory References, (d) SPEC2000 - Faults to other pages.

distribution plots of L shown in Figures 7 (a) and (b). However, an implementation of a replacement algorithm using the lifetime distances depends upon the predictability of $\mathbf{L}$ for a particular virtual memory page. Thus, as the next step, we characterize the predictability of $\mathrm{L}$ for each virtual memory page.

In order to capture this trend, we plot the coefficient of variance of L (coefficient of variance of a sequence is defined as the ratio of standard deviation and the mean of the sequence) for each virtual page. We use this metric instead of the standard deviation since the latter is dependent upon the mean of the sequence and cannot be used as a straightforward yardstick for comparison and/or predictability. In such a graph, points on the $\mathrm{x}$-axis are the individual virtual memory pages and the $y$-axis is the coefficient of variance of $\mathrm{L}$ computed for each page. Figures 10,11 and 12 show the coefficient of variance plots for a few of the NPB and SPEC 2000 applications. Since, we are interested in finding out whether there is predictability in the sequences of lifetime distances observed for each virtual memory page, we would like the coefficient of variance of such a sequence to be as close to 0 as possible. If the coefficient of variance is close to zero, it indicates that the lifetime distance sequence is almost constant and hence we can easily predict the lifetimes of pages. However, it is not necessary that a high coefficient of variance necessarily indicates a lack of predictability. For instance, sequences like those depicted in Figures 8 and 9 may have a high value of coefficient of variance but nonetheless there is a predictable pattern that can be observed. It is also interesting to find that there are applications for which the coefficient of variance for all pages is consistently close to 0 which indicates the underlying predictable nature of the page lifetimes (eg. Figure 10(a)). There are also applications, where we find that a certain subset of pages exhibit coefficient of variance close to 0 and certain others for which it is very high (eg. Figures 10(b), 11(a), 11(b) and 11(c)). There are also applications that do not exhibit either of the above (eg. Figure 12(b)).

Thus far, we have motivated the need for a better replacement algorithm and also looked at the predictability characteristics of the fault parameters (lifetime distances) and observed that there seems to be sufficient predictability for us to investigate better replacement algorithms that will be elaborated upon in the next section. 
(a)

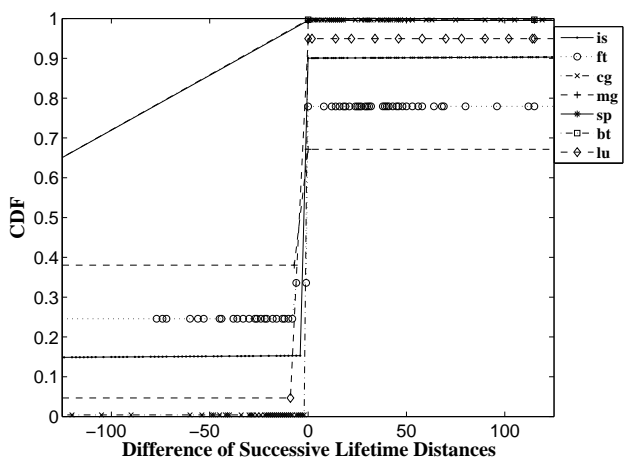

(c)

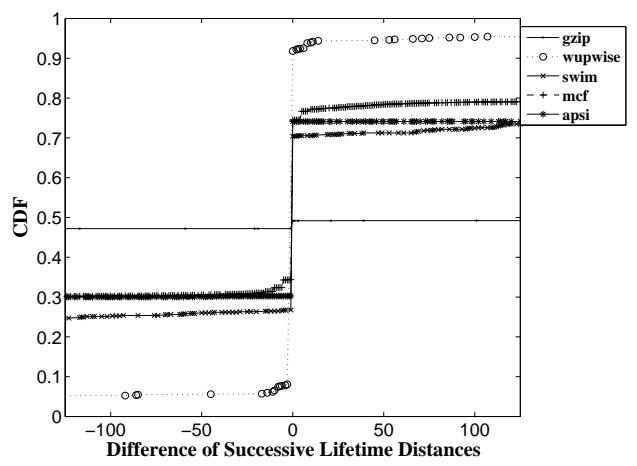

(b)

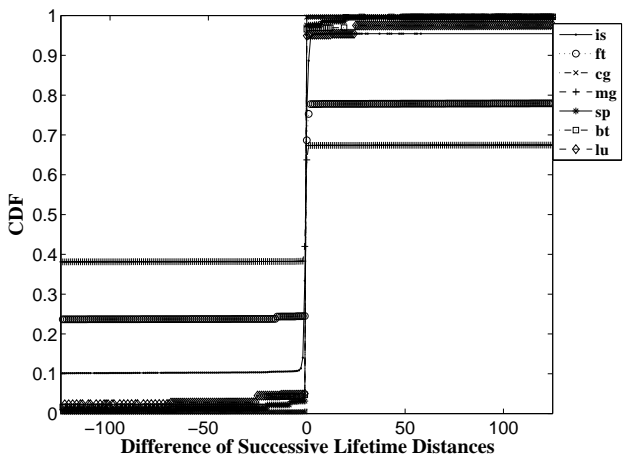

(d)

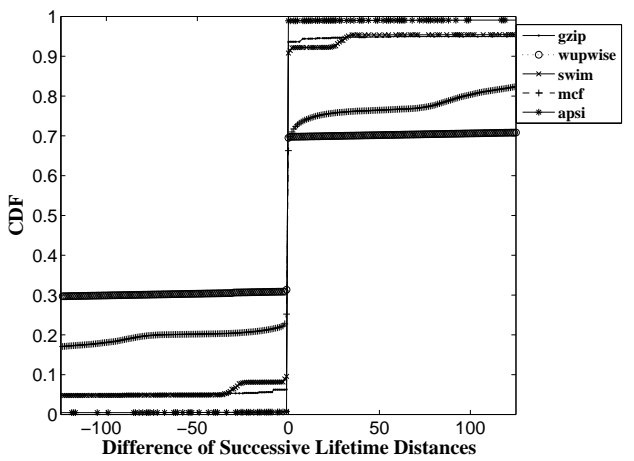

Figure 5: Differences between successive L distances measured as (a) NPB - Total Memory References, (b) NPB Faults to other pages, (c) SPEC2000 - Total Memory References, (c) SPEC2000 - Faults to other pages.

\section{Towards a Better Replacement Algorithm : Predictive Replacement}

In the previous section, we observed that quite a few of the applications exhibited fairly low coefficient of variance of lifetime distances at the individual page granularity, i.e at the individual page granularity there is sufficient regularity of lifetime distances that can be effectively predicted. We also observed that there are also applications in which predictability of lifetime distances for certain virtual memory pages is very low and this indicates the need for some adaptive algorithm that dynamically enables or disables prediction based on the current prediction accuracy. Based on the above observations, we now outline a novel page replacement algorithm. In this approach, the system maintains an additional list that is "approximately" sorted on system predicted values of $\mathbf{L}$ which we call as Dead-List (somewhat of a misnomer, since it keeps track of when a page would become dead in the future rather than currently dead pages!), in addition to the LRU list. Both the LRU and the Dead-Lists are lists of physical memory pages, and hence we would only need to store an extra pointer to traverse the Dead-List. At the time of replacement of pages, the system checks if the head of the Dead-List has expired (i.e, whether or not the system has decided/predicted that a page would not be accessed anymore in this time-interval. The issue of how the system does this prediction is explained a little later in this section.) and if so, it decides to replace that page. Note that since we keep the list approximately sorted on estimated lifetimes, we do not need to look through the whole list. If this list is empty or if the page has not yet been estimated to have expired, then the system defaults to the LRU replacement algorithm. We now present the steps for this predictive replacement algorithm:

- When a page fault occurs on some page $(\mathrm{X})$, we check if the Dead-List is empty, or if the head of the Dead-List is not yet estimated to be dead

- If the above condition is true, we initiate normal LRU replacement algorithm, and delete the page from the Dead-List as well if needed (it may not necessarily be the head of the Dead-List). 
(a)

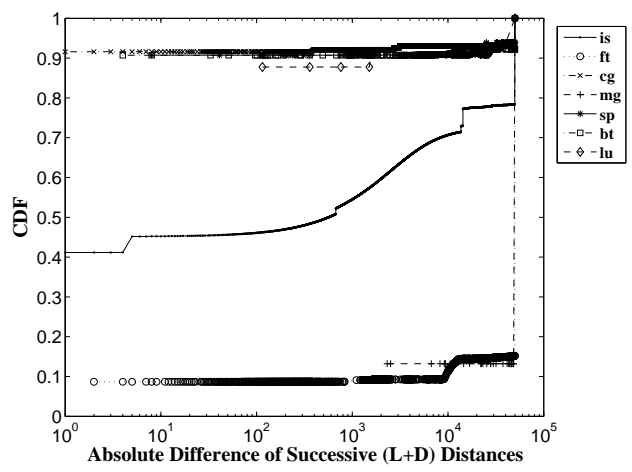

(c)

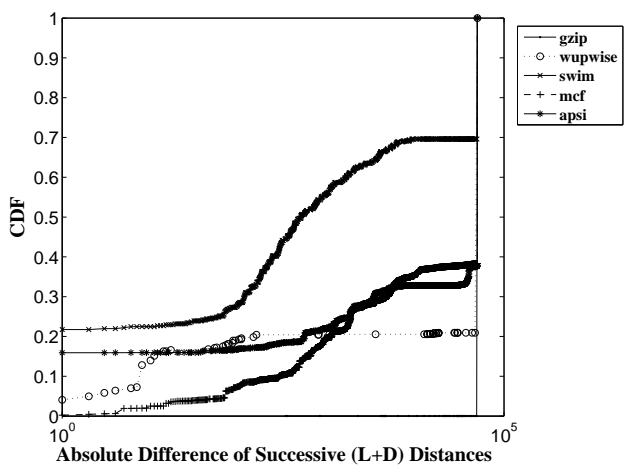

(b)

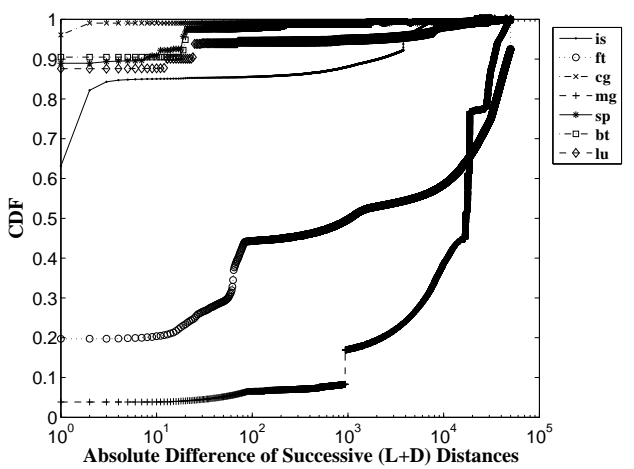

(d)

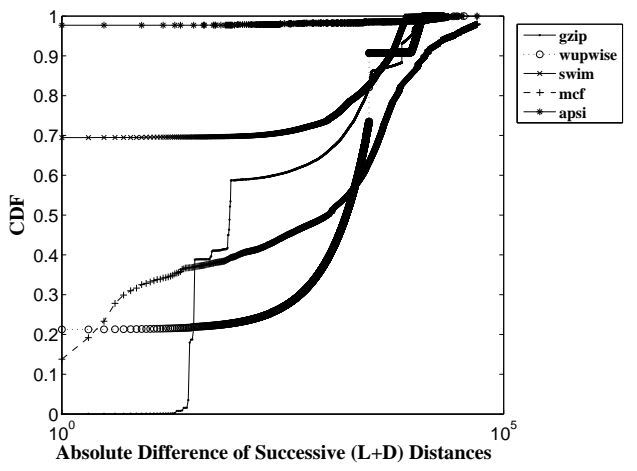

Figure 6: Absolute differences of successive (L+D) distances measured as (a) NPB - Total Memory References, (b) NPB - Faults to other pages, (c) SPEC2000 - Total Memory References, (d) SPEC2000 - Faults to other pages.

- Otherwise, we dequeue the head of the Dead-List and choose that as a candidate for replacement. Note that we need to delete it from the LRU list as well.

- Once the candidate for replacement has been decided, we need to insert the currently faulted page into the sorted Dead-List based on our estimated/predicted value of $\mathbf{L}$ for that page.

Keeping the Dead-list exactly sorted could be a cause of significant overhead, that could have increased the page-fault service time. Consequently, any reductions in page-faults may not have translated to reductions in overall execution time. There are two possible solutions to overcoming this problem. One possible alternative would involve using a min-heap, where the root of the heap would hold the page that is estimated to have the least lifetime, and the other alternative involves keeping the list approximately sorted. The latter scheme involves chaining pages with similar values of estimated lifetime distances in a hash bucket. Note that this kind of scheme also entails one extra pointer per physical memory page since a page cannot be in more than one hash bucket. In the case of a heap-based implementation, the worst-case time complexity of insertion of pages is $\mathrm{O}(\log n)$, and $\mathrm{O}(1)$ for deletion, while the hash-chaining scheme's time complexity is $\mathrm{O}(1)$ for insertion (since pages are always inserted at the tail) and $\mathrm{O}(\mathrm{m}$ ) for deletion of pages, where $n$ is the number of physical memory pages and $m$ is the number of hash chains. Another possible drawback of the heap-based scheme is the need for locking the entire heap during insertion that could prove to be expensive on multi-processor systems, whereas the hash-chaining based scheme involves locking only the appropriate hash chain. In this paper, we have explored the hash-chaining based "approximately" sorted scheme for managing the Dead-List with a fairly small value of $m$ (currently set to 101). Another point to be noted in the above description of the algorithm is the deliberate omission of the prediction mechanism, since that is a parameter that we want to experiment with for good performance. Note that the optimal algorithm would be a perfect predictor, and would replace a page as soon as it becomes dead. Recall that it is possible to measure the lifetime distances accurately only with adequate hardware support and is quite hard to measure it practically in an operating system. Thus, we break up our prediction schemes into two categories: estimation techniques with hardware support and an operating system implementable estimation technique, which are explained in the next two subsections. 
(a)

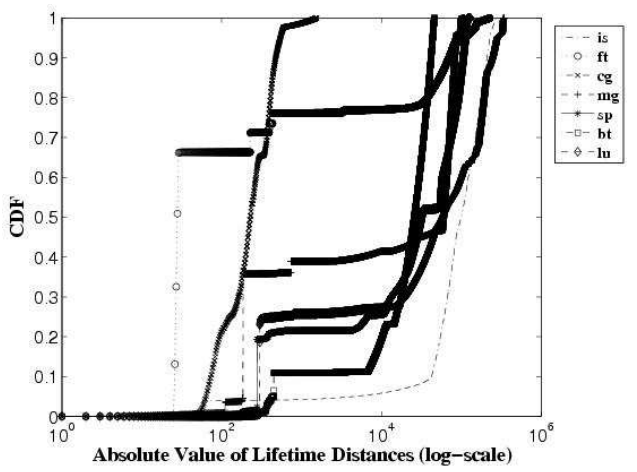

(b)

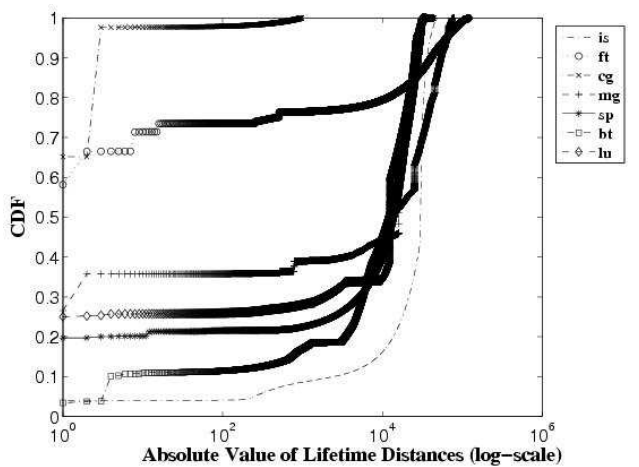

Figure 7: NPB: CDF of L distance measured as (a) References, (b) Faults.

(a)

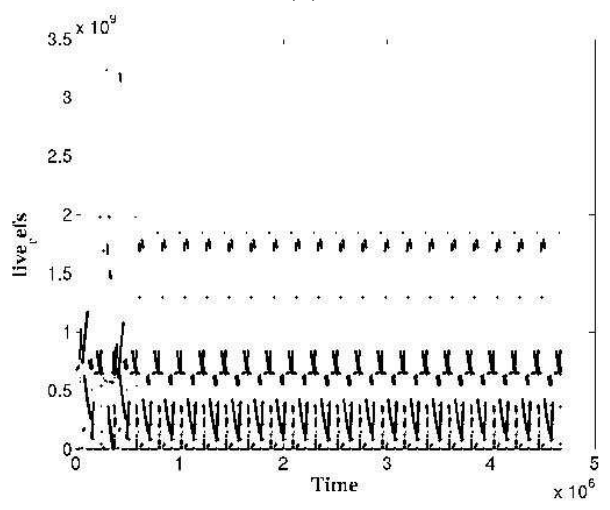

(b)

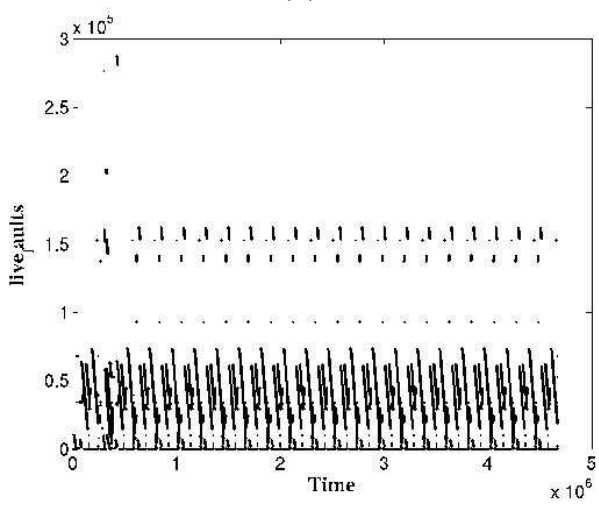

Figure 8: MG: Variation of L distance with time measured as (a) References, (b) Faults.

\subsection{Estimation Techniques with Hardware Support}

With appropriate hardware support, we can keep track of and measure lifetime distances of virtual memory pages using which we have experimented with simple prediction schemes. In all these experiments, the metric that we have used to compare performance is the normalized page-faults when compared to the base LRU scheme. The simulation framework that we have built upon valgrind, maintains two global counters, one which increments on every memory reference (G1) and the other which increments on every page-fault (G2). In addition, each virtual memory page has a set of four counters associated with it which records the following information,

- Timestamp of the last access to that page.(C1)

- Page-Fault Counter at the time of the last access to that page.(C2)

- Timestamp of the previous page-fault to that page.(C3)

- Page-Fault Counter at the time of the previous page-fault to that page.(C4)

A possible concern that might arise in this regard is the storage and access efficiency costs for these counters. A possible solution to this problem is to store these counters in the unused bits of a page-table entry for a virtual memory page, that can also be subsequently cached in the TLB after the first access to the page. On each access to a page (whether it be a hit or a miss) the G1 counter is incremented, and the G2 counter is incremented only on a miss. On every hit access to a page, counters $\mathrm{C} 1$ and $\mathrm{C} 2$ are updated to store the latest values of G1 and G2 respectively. On every page-fault/miss, counters C3 and C4 are updated to store the latest values of G1 and G2 respectively. At 
(a)

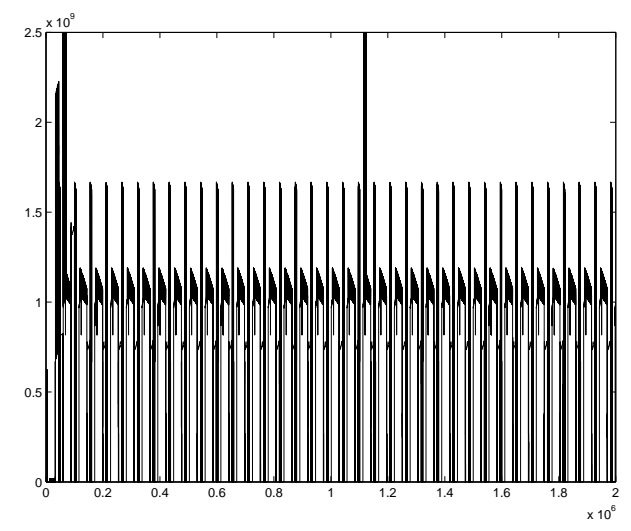

(b)

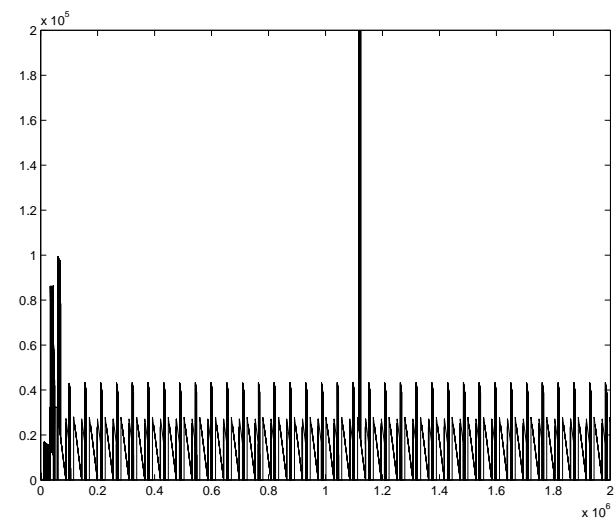

Figure 9: SP: Variation of L distance with time measured as (a) References, (b) Faults.

(a)

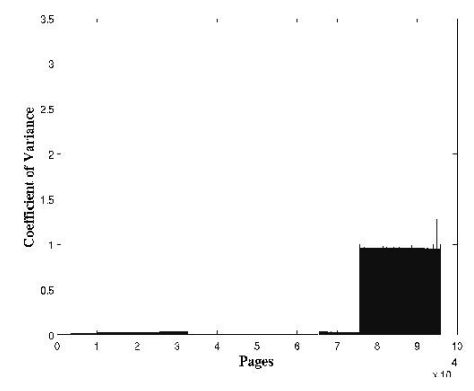

(b)

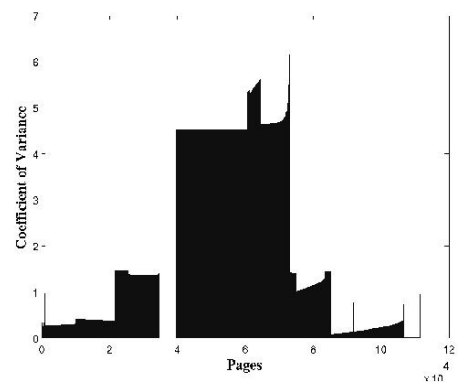

(c)

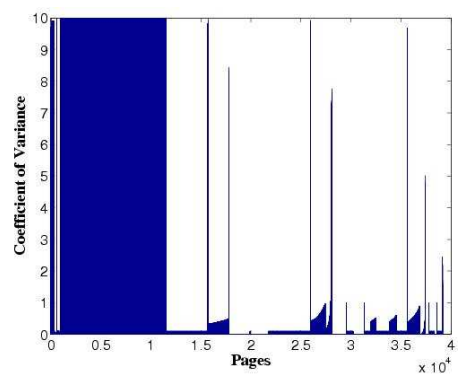

Figure 10: Coefficient of Variance of L for each page (a) IS, (b) MG, (c) SP.

the time of a page-fault (miss), the system can now measure L both in terms of memory references $(\mathrm{L}=\mathrm{C} 1-\mathrm{C} 3)$ and in terms of number of page-faults to other pages $(\mathrm{L}=\mathrm{C} 2-\mathrm{C} 4)$. In all the schemes that are described below, the system uses the measured value of the lifetime distance to predict the next lifetime distance for the page. Once, the prediction is done, we insert the currently faulted page into the appropriate bucket of the Dead-List based on this estimated value. For the remainder of the discussion, we only consider the lifetime distances measured in terms of the number of page-faults to other pages. The characterization experiments in Section 4 indicated the high predictability of Lifetime Distances. In particular, Figures 4 (b), (d) and Figures 5 (b), (d) show that a majority of the differences between successive lifetime distances of a virtual memory page is within a bounded range (around 10), thus indicating that simple variants of a Last-Value predictor would be sufficient in estimating lifetime distances fairly accurately.

- Static variant of Last Value Prediction (Last Static k): In this scheme, if a page's L value was measured to be $\mathrm{L}_{i}$ at the time of a page-fault, we predict that the next lifetime of the page using this scheme as, $\mathrm{L}_{i+1}=\mathrm{L}_{i}$ $+k$, where $k$ is a static constant. For the following set of experiments, we have experimented with 5 different $k$ values, namely $-10,-5,0,5$, and 10 . These values were selected based on the observations made in Figures 5 (b) and (d). Note that a value of 0 for this constant is exactly equivalent to a Last-value predictor, while a positive value of this constant implies a conservative approach towards estimating lifetimes.

- Adaptive Variant of Last Value Prediction (Last-Dynamic): This scheme tries to overcome the previous technique's limitation by trying to reduce the number of predictions made based on observed accuracy of the predictions. In this technique, we associate a state with each virtual memory page that can assume 3 values, namely $\mathrm{S}_{\text {init }}, \mathrm{S}_{\text {transient }}$ and $\mathrm{S}_{\text {pred }}$. The key idea in this algorithm is to disable prediction, unless the state associated with the virtual memory page is equal to $S_{p r e d}$. The algorithm uses a simple 3-state machine and works as follows on a page fault:

- If the state associated with the currently faulted page is $\mathrm{S}_{\text {init }}$, and if the difference $\left(L_{\text {diff }}\right)$ between the last two observed lifetime distances is less than or equal to a threshold $\left(L V_{\text {thresh }}\right)$, it sets the state for the 
(a)

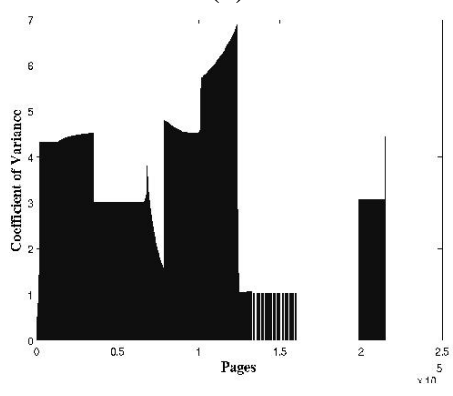

(b)

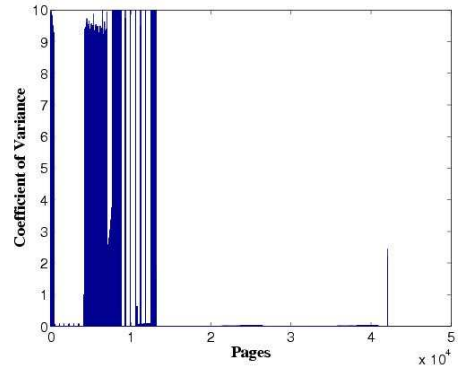

(c)

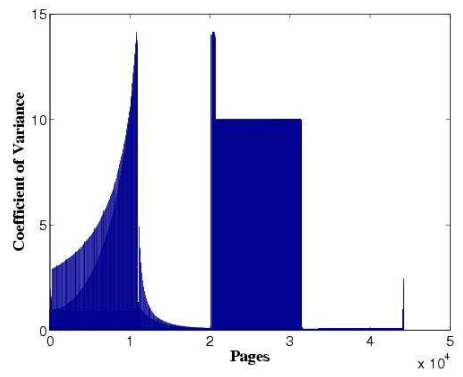

Figure 11: Coefficient of Variance of L for each page (a) FT, (b) BT, (c) LU.

(a)

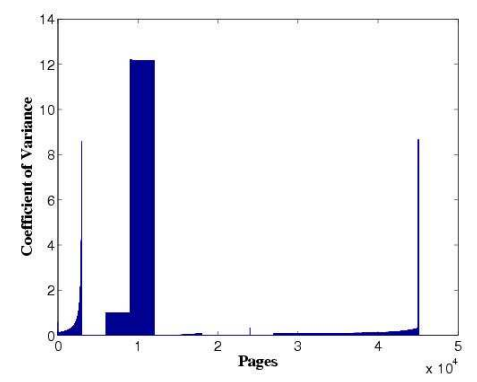

(b)

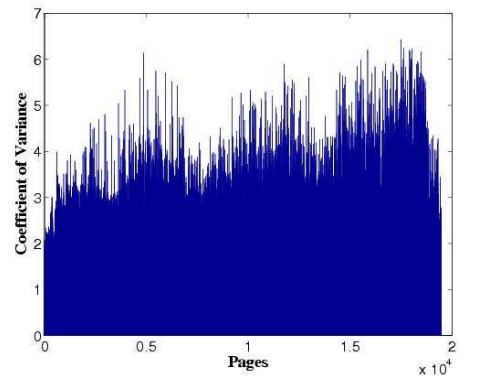

(c)

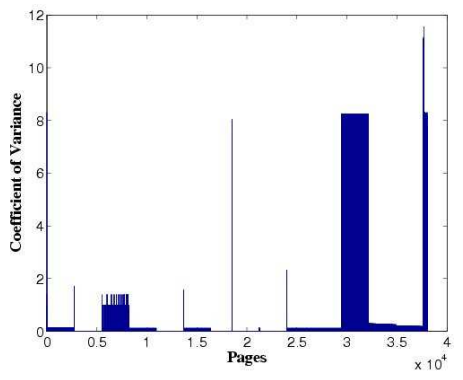

Figure 12: Coefficient of Variance of L distance for each page (a) WUPWISE, (b) MCF, (c) APSI.

currently faulted page to $\mathrm{S}_{\text {transient }}$.

- If the state associated with the currently faulted page is $\mathrm{S}_{\text {transient }}$, and if the difference $\left(L_{\text {diff } f}\right)$ between the last two observed lifetime distances is less than or equal to $L V_{t h r e s h}$, it sets the state for the page to $\mathrm{S}_{\text {pred }}$, and estimates the lifetime of this page as, $\mathrm{L}_{i+1}=\mathrm{L}_{i}+\mathrm{L}_{\text {diff }}$. Otherwise, if the difference $\left(L_{\text {diff }}\right)$ is greater than $L V_{t h r e s h}$, it sets the state of the page to $\mathrm{S}_{\text {init }}$, and disables prediction.

- If the state associated with the currently faulted page is $S_{\text {pred }}$, and if the difference $\left(L_{\text {diff }}\right)$ between the last two observed lifetime distances is less than or equal to $L V_{\text {thresh }}$, it estimates the lifetime of this page as, $\mathrm{L}_{i+1}=\mathrm{L}_{i}+\mathrm{L}_{\text {diff }}$. Otherwise, if the difference $\left(L_{d i f f}\right)$ is greater than $L V_{\text {thresh }}$, it sets the state of the page to $\mathrm{S}_{\text {transient }}$ and disables prediction.

Associating multiple states allows for disabling prediction during temporary bursts and/or sequences where predictability is poor. A critical parameter in the above algorithm is the value of the threshold $\left(L V_{t h r e s h}\right)$, since it determines how aggressive or conservative a scheme is. Choosing a small value for this threshold will allow for very few predictions (conservative), and choosing a large value could potentially allow more prediction based replacements (aggressive). We find that different applications have different ranges of thresholds over which good performance is achieved, as will be shown in the next section. However, the job of determining what are best thresholds for a particular application, memory configuration and relating it to the application characteristics is beyond the scope of this paper and is the focus of our future efforts.

- EELRU: In [SKW99], authors propose an adaptive replacement algorithm that uses a simple online cost-benefit analysis to guide its replacement decision, that is considered to be one of the state-of-art algorithms towards addressing the performance shortcomings of LRU. Hence, we have also compared the performance of our schemes with EELRU in the subsequent evaluations. 


\subsection{OS-Implementable Estimation Technique}

In this scheme, the OS needs to keep a counter (G1) that keeps track of the number of page-faults that have been incurred by the application. On each page-fault, this counter (G1) needs to be incremented. In addition, we need to associate a counter for each virtual page $(\mathrm{C} 1)$, (likewise, this can be stored in the unused bits of the page-table entry after suitable encoding) that is updated whenever a page-fault occurs on that page, i.e we set $\mathrm{C} 1$ to the latest value of $\mathrm{G} 1$ at the time of a page-fault to a page. At the time of subsequent page-faults to the same page, we can now estimate $\mathbf{L}+\mathbf{D}$ as G1-C1. The in-kernel scheme that we propose uses this value to estimate lifetime distance of the page, that we denote as DP-Approx.

- DP-Approx: is a novel replacement algorithm that uses exponential averaging to estimate the lifetime distance. As was mentioned earlier, since the operating system does not get control over individual memory references, any in-kernel approximation of these replacement algorithm needs to make use of OS-visible events like pagefaults and replacements. Therefore, in this technique we start out by estimating the lifetime distance as $\mathbf{L}+\mathbf{D}$, and we subsequently use exponential averaging to predict the next lifetime distance as,

$\mathbf{L}_{\text {pred }}^{t+1}=\mathrm{a} *(\mathrm{~L}+\mathrm{D})_{\text {measured }}^{t}+(1-\mathrm{a}) * \mathrm{~L}_{\text {pred }}^{t}$. Unless otherwise stated, we fix the value of the parameter "a" as 0.5 , which means that we give equal weights to the current measurement and previously estimated lifetimes. One may question that relying on the parameter "a" may reduce the chance of a successful implementation, but we believe that it is possible to build a more sophisticated scheme, where the value of this parameter "a" can be determined dynamically. Please note that our intent in this paper is to demonstrate a proof-of-concept strategy that can be practically realized without too much overheads, and determining the best "a" value automatically, itself is an interesting research topic that we wish to address in the future.

\subsection{Results with Predictive Replacement Techniques}

Figure 13 plots the normalized page-faults for the static and adaptive variants of the last-value prediction based replacement algorithms for the applications, when compared to the base LRU replacement algorithm. The results for the Last static schemes that are shown in Figure 13 have the static parameter set to the following values $(-10,-5,0$, +5 and +10 ). The results for the best performing adaptive scheme (Last dynamic at a fixed value of the threshold) are also shown in Figure 13. The threshold values for each of the application for which the performance was relatively the best is summarized in Table 2. From Table 2, we observe that applications for which the Last static schemes performed well require a higher threshold for the adaptive schemes to show benefits. This can be attributed to the fact that a higher threshold value lends itself to an aggressive algorithm that predicts more often, which in turn is good for such applications as demonstrated by the good performance of the static algorithms. Analogously, applications that perform poorly with the Last static algorithm due to a high number of potentially incorrect predictions require a low value of threshold that lends itself to a conservative algorithm that predicts less often. An interesting area of research that has not been addressed here is the design of a self-tuning, adaptive algorithm that adjusts the thresholds dynamically without manual assignment, which is the focus of our future efforts. As we pointed out earlier, any replacement algorithm that predicts lifetime distances incorrectly may worsen the performance of the application, since it may cause more page-faults by replacing a page ahead of when it actually became dead. From Figure 13, we can observe that the static variants of the predictive algorithm can significantly out-perform LRU in six of the twelve applications (IS, CG, BT, WUPWISE, MCF, SWIM), but can also degrade the performance sometimes quite significantly in the remaining six applications. It is also clear that the adaptive algorithm out-performs LRU in all the applications, thus indicating that the adaptation ensures that the performance never gets degraded badly. It must also be noted that the performance of the adaptive schemes are better than their static variant counterparts in ten of the twelve applications considered and within a factor in the remaining two applications (IS, MCF), which is also indicative that the dynamic scheme adapts itself better, and resorts to using prediction judiciously. Figure 14 plots the normalized invocation counts of the predictive replacement algorithm and the LRU replacement algorithm which is essentially the number of times a particular algorithm was invoked for replacement. In this graph, the five bars for each application denotes the five different schemes for which we have shown results thus far (i.e four static variants, one adaptive scheme). This graph serves to reinforce the fact that the adaptive scheme resorts to using predictionbased replacement as sparingly as possible without worsening the performance. In summary, we find that for all the applications considered an adaptive prediction-based algorithm always performs better than LRU in terms of reductions in number of page-faults, sometimes by as much as $78 \%$. 
(a)

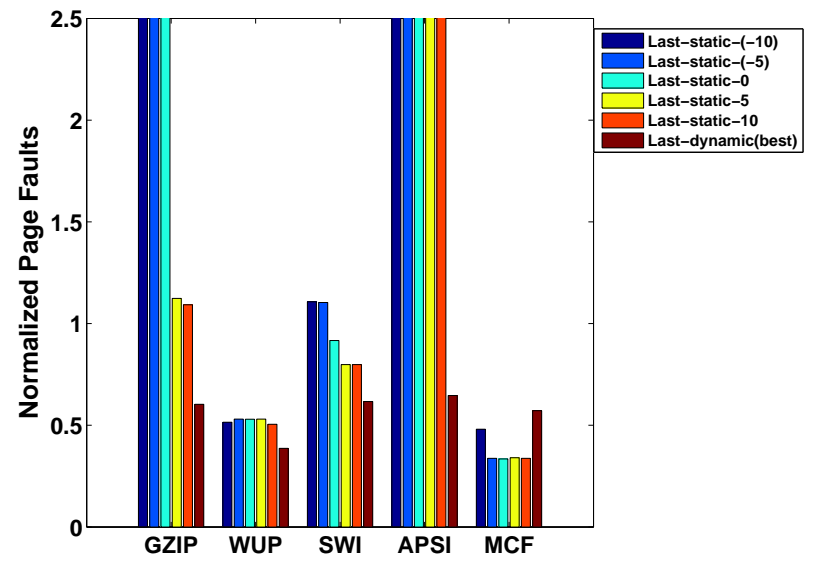

(b)

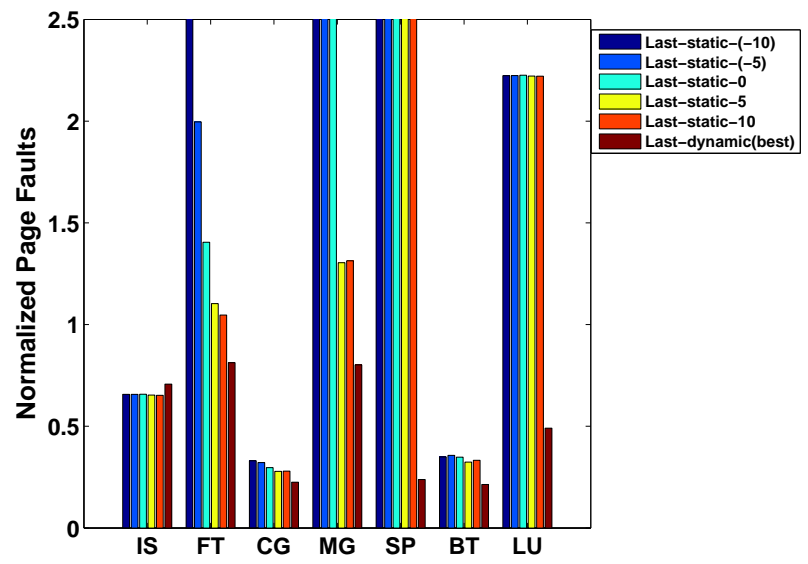

Figure 13: Normalized page-fault counts of the replacement algorithm for (a) SPEC 2000, (b) NPB 2.3 with respect to perfect LRU scheme.

Table 2: Threshold values of applications.

\begin{tabular}{|l|r|l|r|}
\hline NPB 2.3 Application & Threshold & SPEC Application & Threshold \\
\hline IS & 8000 & GZIP & 30 \\
\hline FT & 15 & WUP & 4000 \\
\hline CG & 800 & SWIM & 4000 \\
\hline MG & 30 & MCF & 10000 \\
\hline SP & 60 & APSI & 60 \\
\hline BT & 45 & & \\
\hline LU & 60 & & \\
\hline
\end{tabular}

\subsection{Comparison with EELRU}

[SKW99] proposes an adaptive replacement algorithm (EELRU) that uses the same kind of recency information as LRU and using a simple online cost-benefit analysis, they demonstrate that their algorithm out-performs LRU in the context of virtual memory systems. The basic intuition behind the EELRU technique is that, when the system notices that a large number of pages are being touched in a roughly cyclic pattern that is larger than main memory it diverges from LRU since such a pattern is known to be a worst-case scenario for LRU. In order to detect such situations, the system needs to track the number of pages that were touched since a page was last touched, which is exactly the same kind of information that LRU maintains, but EELRU maintains it for both resident and non-resident pages. Once such a situation is detected, they apply a fall-back replacement algorithm that evicts either the least-recently used page or a page from a pre-determined recency position. In the context of virtual memory systems, the EELRU approach is considered to be one of the state-of-art approaches towards improving the performance of LRU, and hence, we wished to compare the performance of our schemes with EELRU. Note that EELRU is also a simulation-based approach, and no practical approximation of it has been demonstrated thus far in an operating system. Hence, the comparison is done only with the hardware-based simulation techniques that we have proposed thus far (see Section 5.1).

The first two bars for each application in Figure 15 shows the normalized page fault counts for the best performing prediction-based replacement algorithm and EELRU with respect to perfect LRU, while the last bar shows the relative reduction in page faults of the prediction-based replacement algorithm over EELRU Therefore, greater the last bar, the better the prediction-based algorithm performs when compared to EELRU. From Figure 15, it is clear that both the prediction-based and EELRU replacement algorithms out-perform LRU for all the applications. Further, we also notice that the best performing predictive replacement schemes outperforms EELRU in nine out of the twelve applications that we tested against, namely FT (17.49\%), CG (3.84\%), MG (16.19\%), SP (75.56\%), BT (78.18\%), LU (50.43\%), WUPWISE (35.36\%), SWIM (38.03\%) and APSI (33.78\%), where the percentages in parentheses indicate the reduction in number of page-faults compared to the EELRU scheme. With the exception of GZIP, where EELRU performs dramatically better than any of our prediction schemes, we find that on the average, our scheme generates 
(a)

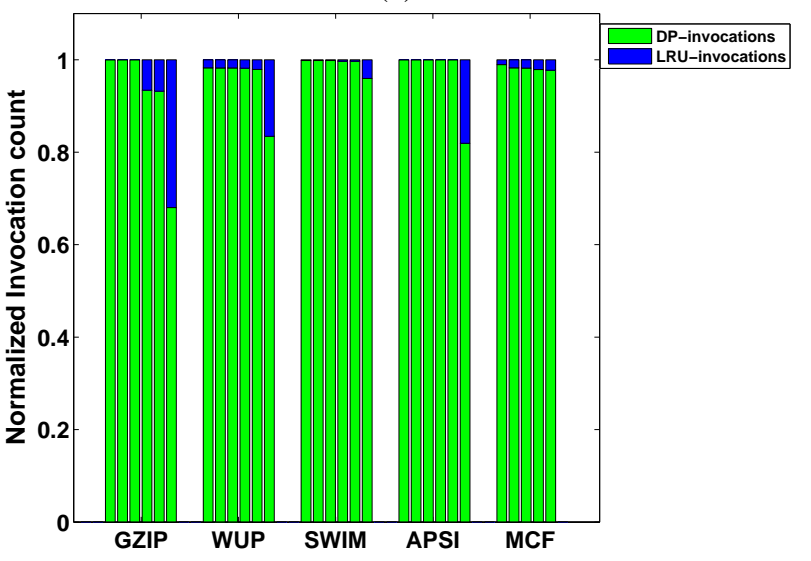

(b)

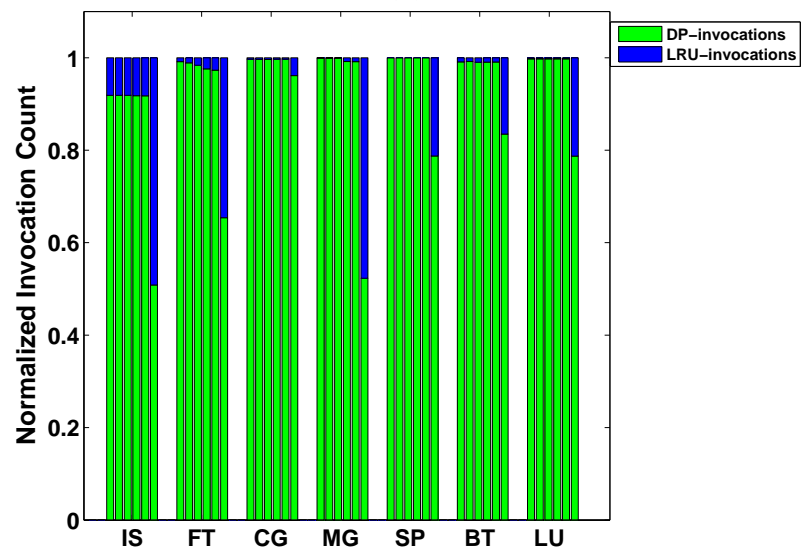

Figure 14: Normalized invocation counts of the replacement algorithm for (a) SPEC 2000, (b) NPB 2.3.

(a)

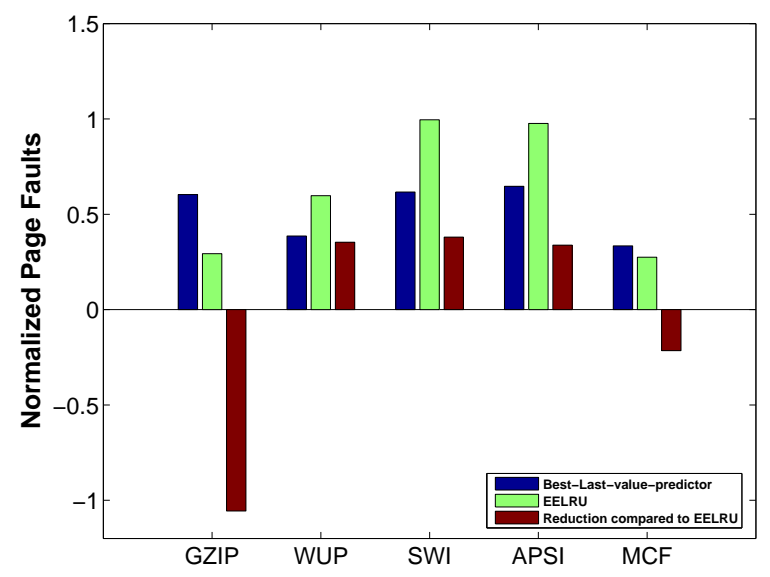

(b)

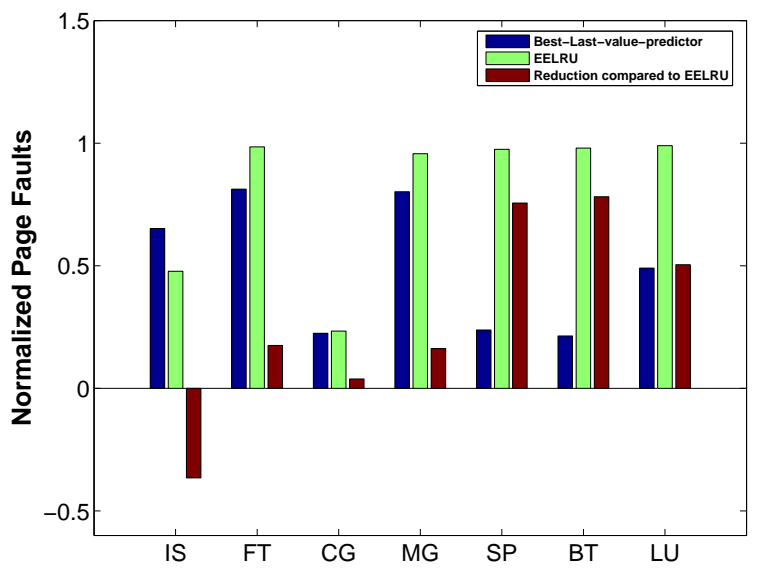

Figure 15: Comparison of the best prediction-based replacement algorithm with EELRU for (a) SPEC 2000, (b) NPB 2.3.

around 15\% lower page-faults than EELRU over all the applications (around 26\% lower page-faults than EELRU over all applications except GZIP). It must be remembered that the predictive algorithm needs sufficient history to start prediction, and in two of the three applications (IS, GZIP) where EELRU performs better, quite a few pages are accessed exactly once which does not allow the prediction based replacement algorithm to start replacing such pages pro-actively.

\subsection{Performance of DP-Approx}

As discussed in Section 5.2, DP-Approx is an in-kernel practical, realizable implementation of the prediction-based replacement techniques. The kernel uses exponential averaging of the measured $(\mathbf{L}+\mathbf{D})$ distances to estimate the lifetime distances of virtual memory pages. For a fair evaluation of this scheme, we show the reduction in the number of page-faults over the actual number of page-faults reported by the Linux kernel when running the application natively. We augmented the Linux 2.4.20 kernel with a new system call (getrusage2), along the lines of an existing system call (getrusage) that returns the number of cold and warm misses/faults. The experimental data for this study was collected on a uni-processor Xeon-based machine running the modified Linux 2.4.20 kernel that was instructed to use a specified amount of main memory through its command line options specified in the bootloader, and is the average over five runs of the application. Figure 16 plots the normalized reduction in the number 
of page faults using the DP-Approx technique in comparison to the Linux 2.4.20 kernel's page-faults. It is clear from Figure 16 that the DP-Approx technique outperforms the kernel's replacement algorithm in all but one application (APSI) by reducing page-faults by as much as $56 \%$. We find that on the average, the DP-Approx scheme gives around $14 \%$ lower page-faults than the kernel's replacement algorithm over all the applications (18\% lower page-faults than the kernel's replacement scheme over all applications except APSI).

(a)

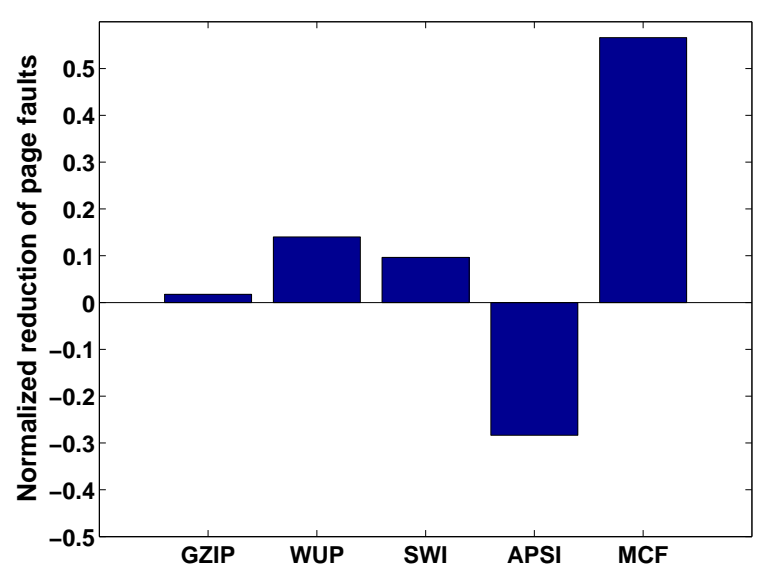

(b)

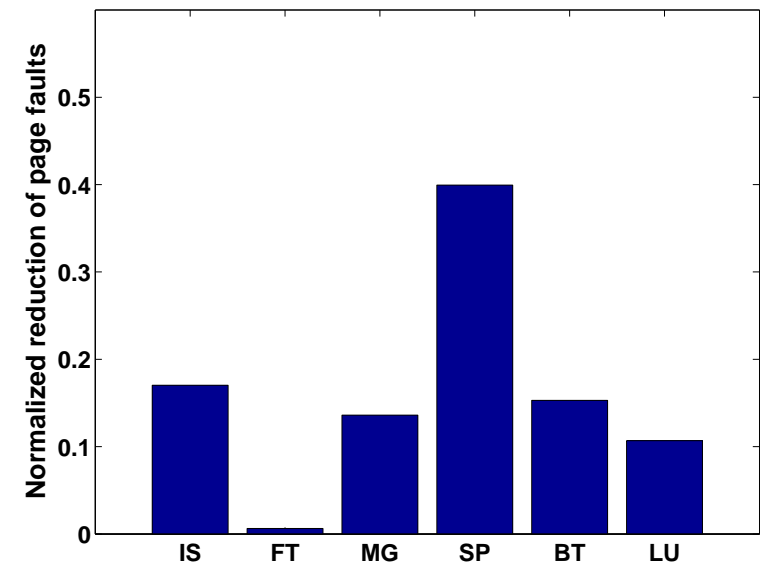

Figure 16: Normalized page-fault reduction of DP-Approx algorithm in comparison to Linux kernel 2.4.20 execution for (a) SPEC 2000, (b) NPB 2.3.

\subsection{Sensitivity Analysis}

While the techniques that we have proposed thus far, yielded significant reductions in page-faults compared to LRU, it must be remembered that most of these schemes (with the exception of DP-Approx) cannot translate to an in-kernel implementation without hardware support, due to the inability of the operating system to keep track of individual memory references. In the DP-Approx technique, we approximate the lifetime distances by exponentially averaging observed $(\mathbf{L}+\mathbf{D})$ distances in the operating system. Recall from the earlier discussion that this technique depended on the exponential averaging factor "a", and in the next set of experiments, we wanted to study the impact on the performance by varying this parameter. In Figures 17 (a) and 18 (a), we plot the normalized page-faults incurred by application with the DP-Approx replacement technique over that of LRU. Although such a comparison is not really fair, since one is a scheme that can be implemented, and the other can at best be approximated, we wanted to see if any trends can be observed that can help in designing a self-tuning online algorithm. We observe that in most of the applications the value of "a" is critical for good performance. In Figures 17 (b) and 18 (b), we plot the prediction accuracy of the exponential averaging scheme for the different values of "a" and find that in almost all the cases, we ended up under-predicting lifetime distances which sort of reflects on why the performance of this replacement algorithm is not so much better than LRU. The reason for the poor performance due to under-prediction is because of the increased page-faults that the application incurs. Note, that a large number of over-predictions may also not perform better than LRU since we may not be aggressive enough to evict pages as soon as they become "dead". In fact, we can see that in applications like IS, MG, MCF and WUPWISE where this scheme performs better than LRU, the percentage of over-predictions is higher than the rest. This indicates that any approximation scheme needs to track the lifetime distances quite accurately for good performance.

\section{Conclusions and Future Work}

In this paper, we have presented a novel technique of tracking application's virtual memory access pattern in the operating system for pro-active memory management by replacing virtual memory pages as soon as they become dead. The contributions of this work can be summarized as follows: 
(a)

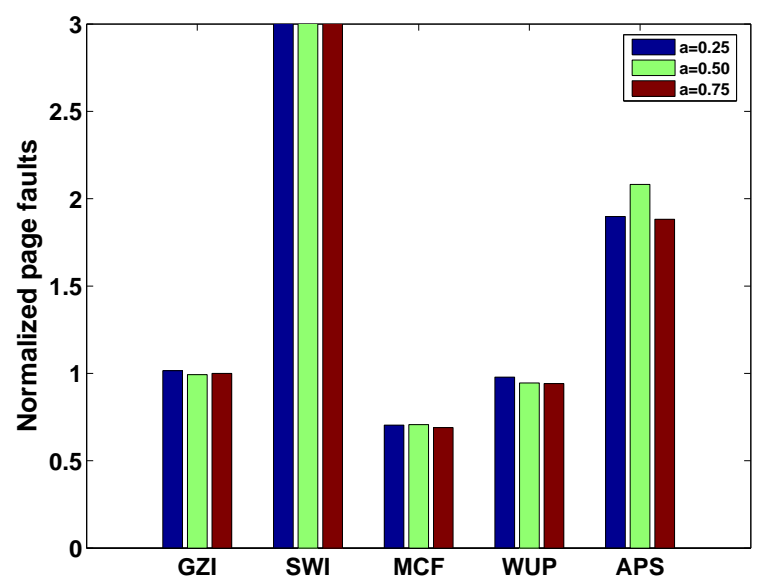

(b)

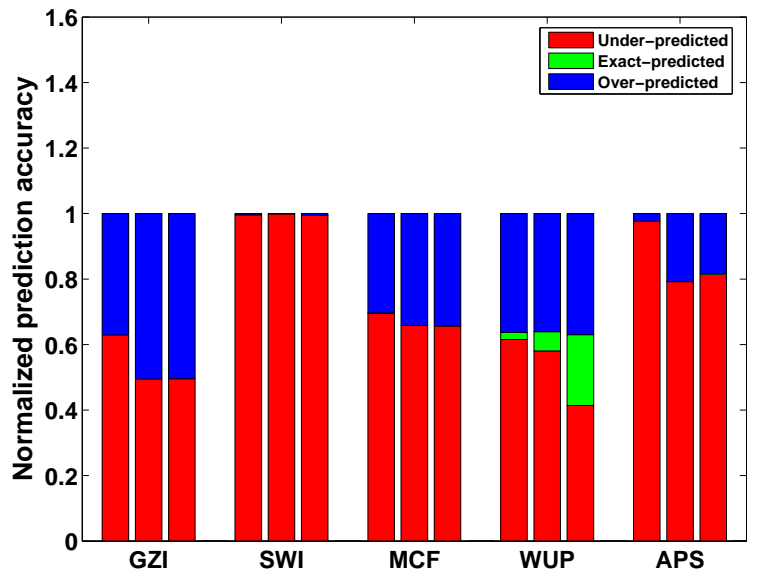

Figure 17: SPEC 2000 (a) Sensitivity of DP-Approx to parameter “a”, (b) Prediction accuracy.

(a)

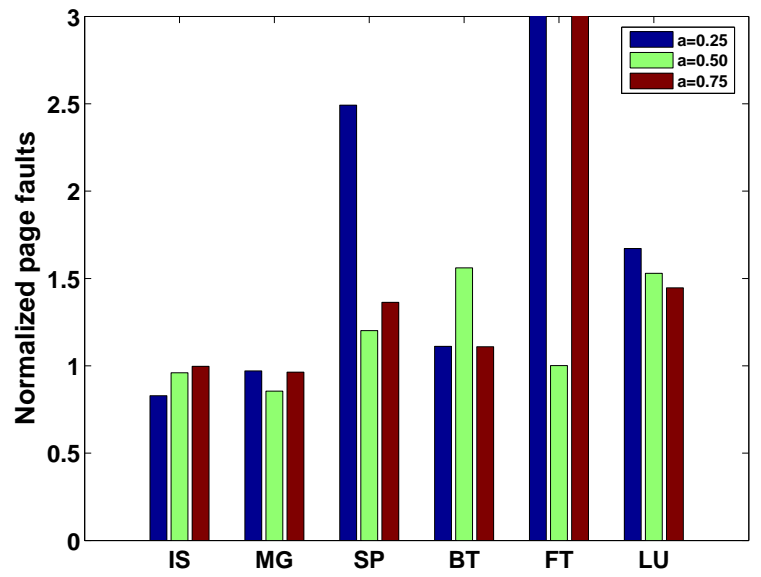

(b)

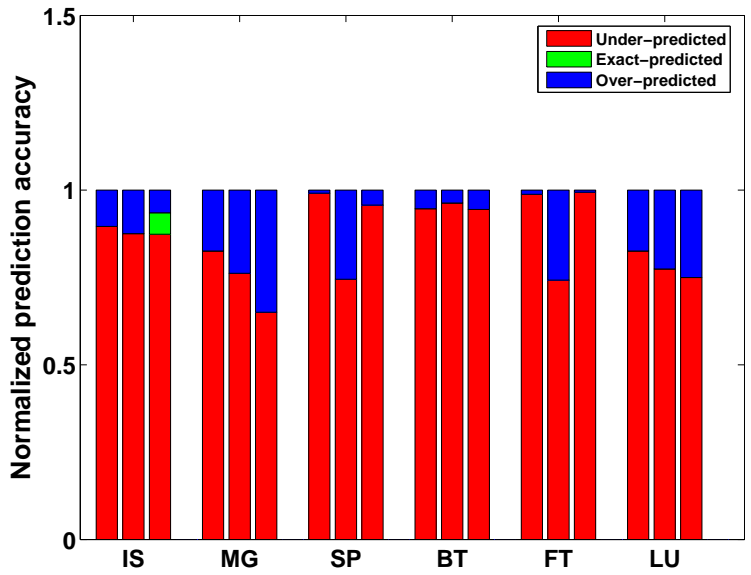

Figure 18: NPB 2.3 (a) Sensitivity of DP-Approx to parameter “a”, (b) Prediction accuracy.

- Demonstrating the sub-optimal performance of LRU-like replacement algorithms for scientific applications' access patterns from the application characteristics and fault parameters perspective and demonstrating the fact that LRU-like replacement algorithms hold onto virtual memory pages long after they are "dead".

- Characterizing the predictability of the fault parameters from an application's perspective.

- Using these parameters in conjunction with simple predictors (variants of Last-value predictors) to design a novel set of replacement algorithms.

- Evaluating the performance of these replacement algorithms on a set of 12 different memory intensive applications drawn from the NAS and SPEC 2000 application suite and concluded that a prediction-based replacement algorithm can significantly out-perform LRU by yielding as much as $78 \%$ reduction in the number of pagefaults. On the average, a prediction-based replacement scheme yields around $48 \%$ reduction in page-faults in comparison to LRU.

- Evaluating and comparing the performance of our techniques with EELRU that is considered to be one of the state-of-art algorithms towards improving performance of LRU in the context of virtual memory systems and demonstrate that our predictive replacement schemes can reduce number of page-faults over EELRU in 9 of the 12 memory intensive applications with which we experimented by as much as $78 \%$. On the average, the predictive replacement schemes yield around 15\% lower page-faults than EELRU. 
- Designing and implementing a novel in-kernel approximation algorithm that can estimate lifetime distances using just the parameters that an operating system can measure. On the average, this algorithm yields around 14\% lower page-faults than a Linux 2.4.20 kernel's replacement algorithm and by as much as $56 \%$ reduction in the number of page-faults. This can serve as a much better alternative to approximate LRU or not recently used replacement algorithms (note that schemes such as LRU or EELRU are not implementable in practice) that are typically implemented in the kernel.

We do realize that there is a need for a more exhaustive and detailed sensitivity analysis to confirm that the replacement algorithms work well across the entire spectrum of memory configurations for all the applications. Many of the proposed schemes also depend upon parameters that needs to be tuned for good performance. For instance, the adaptive variant of the Last-Value predictor based replacement algorithm relies on the threshold value, and DPApprox relies upon the exponential averaging constant for good performance. Consequently, an interesting avenue of research that we have not addressed in this paper is relating the application characteristics to auto-select parameters or a self-tuning algorithm that auto-tunes the parameters for good performance. We also have not explored the effects of multi-programming and OS implementation issues (like memory space needed for prediction), which are the focus of our future research agenda. Another interesting aspect that needs investigation is a prediction-based prefetching mechanism similar to the replacement techniques proposed here. We also plan to design and implement the optimal predictor based replacement algorithm and compare its performance with our prediction based replacement algorithm to get an idea of how much better we could perform. In the future, we also plan to incorporate compile-time information on future access patterns that can be used in conjunction with the run-time based prediction schemes that we have proposed.

\section{References}

$\left[\mathrm{BBB}^{+}\right.$91] D. H. Bailey, E. Barszcz, J. T. Barton, D. S. Browning, R. L. Carter, D. Dagum, R. A. Fatoohi, P. O. Frederickson, T. A. Lasinski, R. S. Schreiber, H. D. Simon, V. Venkatakrishnan, and S. K. Weeratunga. The NAS Parallel Benchmarks. The International Journal of Supercomputer Applications, pages 63-73, Fall 1991.

[BCR96] R. Bordawekar, A. Choudhary, and J. Ramanujam. Automatic Optimization of Communication in Compiling Out-ofCore Stencil Codes. In Proceedings of the 10th ACM International Conference on Supercomputing, pages 366-373, Philadelphia, PA, 1996. ACM Press.

[Bel66] L. A. Belady. A Study of Replacement Algorithms for Virtual Storage Computers. IBM Systems Journal, 5:78-101, 1966.

[BM00] A. D. Brown and T. C. Mowry. Taming the Memory Hogs: Using Compiler-Inserted Releases to Manage Physical Memory Intelligently. In Proceedings of the 2000 Symposium on Operating Systems Design and Implementation, pages 31-44. USENIX Association, 2000.

[BM04] S. Bansal and D. Modha. CAR: Clock with Adaptive Replacement. In Proceedings of the USENIX Conference on File and Storage Technologies, pages 187-200. ACM Press, 2004.

[BTC94] R. Bordawekar, R. Thakur, and A. Choudhary. Efficient Compilation of Out-of-core Data Parallel Programs. Technical Report SCCS-622, Syracuse University, 1994.

[CE97] M. Cox and D. Ellsworth. Application-controlled Demand Paging for Out-of-Core Visualization. In IEEE Visualization, pages 235-244, 1997.

[CFKL95] P. Cao, E. W. Felten, A. R. Karlin, and K. Li. A Study of Integrated Prefetching and Caching Strategies. In Proceedings of the 1995 ACM SIGMETRICS Joint International Conference on Measurement and Modeling of Computer Systems, pages 188-197. ACM Press, 1995.

[CFL94] P. Cao, E. W. Felten, and K. Li. Implementation and Performance of Application-Controlled File Caching. In Proceedings of Operating Systems Design and Implementation (OSDI) Conference, pages 165-177, 1994.

[CG99] F. W. Chang and G. A. Gibson. Automatic I/O Hint Generation Through Speculative Execution. In Proceedings of the Operating Systems Design and Implementation (OSDI) Conference, pages 1-14, 1999.

[Cor69] F. J. Corbato. A Paging Experiment with the Multics System, 1969. Included in a Festschrift published in honor of Prof. P.M. Morse. MIT Press, Cambridge, Mass.

[FC03] K. Fraser and F. W. Chang. Operating System I/O Speculation: How Two Invocations are Faster than One. In Proceedings of the USENIX Annual Technical Conference (General track), 2003.

[GA94] J. Griffioen and R. Appleton. Reducing File System Latency using a Predictive Approach. In Summer USENIX Technical Conference, pages 197-207, 1994.

[GC97] G. Glass and P. Cao. Adaptive Page Replacement based on Memory Reference Behavior. In Proceedings of the 1997 
ACM SIGMETRICS International Conference on Measurement and Modeling of Computer Systems, pages 115-126. ACM Press, 1997.

[HC92] K. Harty and D. R. Cheriton. Application-controlled Physical Memory using External Page-cache Management. In Proceedings of the 5th International Conference on Architectural Support for Programming Languages and Operating System (ASPLOS), pages 187-197, New York, NY, 1992. ACM Press.

[Hen00] J. L. Henning. SPEC CPU2000: Measuring CPU Performance in the New Millennium. IEEE Computer, 33(7):28$35,2000$.

[JS94] T. Johnson and D. Shasha. 2Q: A Low Overhead High Performance Buffer Management Replacement Algorithm. In Proceedings of the Twentieth International Conference on Very Large Databases, pages 439-450, Santiago, Chile, 1994.

[JZ02] S. Jiang and X. Zhang. LIRS: An Efficient Low Inter-Reference Recency Set Replacement Policy to improve Buffer Cache Performance. In Proceedings of the 2002 ACM SIGMETRICS International Conference on Measurement and Modeling of Computer Systems, pages 31-42. ACM Press, 2002.

[KL96] T. M. Kroeger and D. D. E. Long. Predicting File-System Actions From Prior Events. In Proceedings of the USENIX 1996 Annual Technical Conference, pages 319-328, 1996.

$\left[\mathrm{LCC}^{+}\right.$97] D. Lee, J. Choi, H. Choe, S. Noh, S. Min, and Y. Cho. Implementation and Performance Evaluation of the LRFU Replacement Policy. In Proceedings of the $23^{\text {rd }}$ Euromicro Conference, pages 106-111, 1997.

[LFF01] An-Chow Lai, Cem Fide, and Babak Falsafi. Dead-block Prediction and Dead-block Correlating Prefetchers. In Proceedings of the 28th Annual International Symposium on Computer Architecture, pages 144-154. ACM Press, 2001.

[MDK96] T. C. Mowry, A. K. Demke, and O. Krieger. Automatic Compiler-Inserted I/O Prefetching for Out-of-Core Applications. In Proceedings of the 1996 Symposium on Operating Systems Design and Implementation, pages 3-17. USENIX Association, 1996.

[MM04] N. Megiddo and D. S. Modha. Outperforming LRU with an Adaptive Replacement Cache Algorithm. Computer, 37(4):58-65, 2004.

$\left[\mathrm{NMS}^{+} 00\right]$ A. Nanda, K-K. Mak, K. Sugavanam, R. K. Sahoo, V. Soundararajan, and T. B. Smith. MemorIES: A Programmable, Real-Time Hardware Emulation Tool for Multiprocessor Server Design. SIGPLAN Notices, 35(11):37-48, 2000.

[OOW93] E. J. O'Neil, P. E. O'Neil, and G. Weikum. The LRU-k Page Replacement Algorithm for Database Disk Buffering. In International Conference on Management of Data and Symposium on Principles of Database Systems, ACM SIGMOD, pages 297-306, Washington, D.C., 1993.

[PG95] V. Phalke and B. Gopinath. An Inter-Reference Gap model for temporal locality in program behavior. In Proceedings of the 1995 ACM SIGMETRICS Joint International Conference on Measurement and Modeling of Computer Systems, pages 291-300, 1995.

[PGG ${ }^{+}$95a] R. Patterson, G. Gibson, E. Ginting, D. Stodolsky, and J. Zelenka. Informed Prefetching and Caching. In Proceedings of the 15th Symposium on Operating Systems Principles, pages 79-95, Copper Mountain, Colorado, 1995.

$\left[\mathrm{PGG}^{+}\right.$95b] R. H. Patterson, G. A. Gibson, E. Ginting, D. Stodolsky, and J. Zelenka. Informed Prefetching and Caching. In Hai Jin, Toni Cortes, and Rajkumar Buyya, editors, High Performance Mass Storage and Parallel I/O: Technologies and Applications, pages 224-244. IEEE Computer Society Press and Wiley, New York, NY, 1995.

[PKK95] M. Paleczny, K. Kennedy, and C. Koelbel. Compiler support for out-of-core arrays on data parallel machines. In Proceedings of the 5th Symposium on the Frontiers of Massively Parallel Computation, pages 110-118, McLean, VA, 1995.

[SKW99] Y. Smaragdakis, S. Kaplan, and P. R. Wilson. EELRU: Simple and Effective Adaptive Page Replacement. In Proceedings of the 1999 ACM SIGMETRICS International Conference on Measurement and Modeling of Computer Systems, pages 122-133. ACM Press, 1999.

[SN03] J. Seward and N. Nethercote. Valgrind, An Open Source Memory Debugger for x86-linux, 2003. http://developer.kde.org/ sewardj/.

[WGRW93] D. Womble, D. Greenburg, R. Riesen, and S. Wheat. Out of core, out of mind: Practical Parallel I/O. In Scalable Parallel Libraries Conference, pages 10-16, October 1993. 